\title{
Avaliação dos resíduos de serviços de saúde do Grupo A em hospitais de Vitória (ES), Brasil
}

\author{
Evaluation of healthcare wastes service of group A in the hospitals of \\ Vitória (ES), Brazil
}

\section{Saulo Alves Aduan', Florindo dos Santos Braga ${ }^{2}$, Eliana Zandonade 3 , David Salles ${ }^{4}$, Noil Amorim de Menezes Cussiol ${ }^{5}$, Liséte Celina Lange ${ }^{6}$}

\begin{abstract}
RESUMO
Nesta pesquisa, quantificam-se e classificam-se, conforme a Resolução RDC ANVISA no 306/2004, os Resíduos de Serviço de Saúde do Grupo A, gerados em seis hospitais de Vitória (ES), Brasil. Os resíduos acondicionados sem segregação foram separados por grupos e subgrupos, resultando em: 57\% do Grupo D - comum; 41\% do Grupo A - risco biológico; 1,5\% do Grupo B - risco químico e 0,05\% do Grupo E - perfuro-cortantes. O peso específico aparente foi de 106,2 kg.m-3 e a taxa média de geração de resíduos foi 2,68 kg. (leito.ocupado.dia)-1. Ao se segregar os resíduos do Grupo D, a taxa de geração do Grupo A passou a ser 1,15 kg.(leito.ocupado.dia)-1, resultando em 58\% de redução nos custos com incineração. Com a segregação dos Grupos B, D, E e do subgrupo A4, a taxa de geração passou a ser 0,18 kg.(leito.ocupado.dia)-1, com uma redução nos custos com incineração de 93\%.
\end{abstract}

Palavras-chave: gerenciamento de resíduos de serviços de saúde; RSS; classificação de RSS; resíduos; caracterização gravimétrica.

\begin{abstract}
In this research the Health Care Waste of Group A (Resíduos de Serviço de Saúde - RSS), collected from six hospitals in Vitoria (ES), Brazil, was quantified and classified according with ANVISA's Resolution RDC n० 306/2004. The waste was packaged without segregation and afterwards separated by groups and subgroups, with the following results/percentages: 57\% Group D - common; 41\% Group A - biological risk; 1.5\% Group B-chemical risk; and 0.05\% Group E-sharps. The apparent bulk density was $106.2 \mathrm{~kg} / \mathrm{m} 3$. The average production rate of this mixture was $2.68 \mathrm{~kg}$ per occupied bed daily. By separating the mixture of the RSS from group D, the average production rate of Group A changed to $1.15 \mathrm{~kg}$ per occupied bed daily, reducing incineration costs by $58 \%$. By segregating Groups B, D, E and subgroup A4, the average production rate changed to 0.18 $\mathrm{kg}$ per occupied bed per day with a reduction of $93 \%$ in incineration costs.
\end{abstract}

Keywords: healthcare waste management; health care waste; classification of healthcare waste; waste; gravimetric characterization of healthcare waste.

\section{INTRODUÇÃO}

No Brasil, a RDC ANVISA n 306/2004 e Resolução CONAMA $n^{\circ} 358 / 2005$ preconizam que todos os estabelecimentos prestadores de serviços de saúde no país, sejam públicos ou privados, são obrigados a elaborar, implantar e monitorar o seu Plano de Gerenciamento de Resíduos de Serviços de Saúde (PGRSS) (BRASIL, 2004, 2005). Segundo essas resoluções, a segregação consiste na separação dos resíduos, por grupo e subgrupos de classificação, no momento e local de sua geração, de acordo com as características físicas, químicas, radiológicas e biológicas; o estado físico e os riscos envolvidos, demonstrando que essa etapa é uma das mais importantes do gerenciamento dos resíduos de serviços de saúde (RSS). A caracterização e classificação dos RSS são o ponto de partida para a realização de uma coleta, transporte, tratamento e disposição final mais seguros para o homem e o meio ambiente. Os estabelecimentos geradores devem averiguar se o gerenciamento dos RSS está ou não atendendo a essas legislações, principalmente quanto à segregação correta na fonte de geração e destinação final conforme sua classificação.

\footnotetext{
'Mestre em Engenharia Ambiental pela Universidade Federal do Espírito Santo (UFES) - Vitória (ES), Brasil.

${ }^{2}$ Professor Associado do Departamento de Engenharia Ambiental da UFES - Vitória (ES), Brasil. Pós-doutorando pela Universidade Federal de Minas Gerais (UFMG) Belo Horizonte (MG), Brasil.

${ }^{3}$ Professora Associada do Departamento de Estatística da UFES e do Programa de Pós-graduação em Saúde Coletiva da UFES - Vitória (ES), Brasil

${ }^{4}$ Graduando em Ciências Biológicas pela Faculdade Salesiana de Vitória. Técnico de Enfermagem e Membro do Comitê de Gerenciamento de RSS do Centro de Ciências da Saúde da UFES - Vitória (ES), Brasil.

${ }_{5}^{5}$ Doutora em Meio Ambiente pela UFMG; Pesquisadora do Centro de Desenvolvimento da Tecnologia Nuclear - Belo Horizonte (MG), Brasil.

${ }^{6}$ Professora Associada do Departamento de Engenharia Sanitária e Ambiental da UFMG - Belo Horizonte (MG), Brasil.

Endereço para correspondência: Saulo Alves Aduan - Rua Haylton Bassini Junior, 91, apto. 104B - Mata da Praia - $29065-440$ - Vitória (ES), Brasil - E-mail: sauloaduan@gmail.com Recebido: 11/06/12 - Aceito: 28/10/13 - Reg. ABES: 414
} 
Da busca de informações, registros de geração e classificação dos RSS em atendimento à RDC ANVISA n 306/2004 - como taxa de geração de RSS por leito ocupado por dia, peso específico aparente e composição gravimétrica - junto aos órgãos de saúde e de controle ambiental federal, estadual e municipal e em dezesseis hospitais do município de Vitória (ES), Brasil, constatou-se a total inexistência desses dados.

Da literatura consultada sobre gerenciamento de RSS em hospitais, a maioria aponta a segregação incorreta na fonte de geração como o principal fator responsável pelos gastos com transporte e disposição final. A falha mais comum encontrada é o acondicionamento junto dos resíduos dos Grupos D e A. Sobre as taxas de geração, têm-se os trabalhos nacionais de Cussiol, Lange e Ferreira (2001); Salomão, Trevizan e Günther (2004); Souza (2005) e Gil (2007) e, sobre resultados de peso específico aparente, têm-se os estudos de Diaz et al. (2007) e Monteiro et al. (2001).

Os objetivos da pesquisa foram quantificar e classificar, segundo a RDC ANVISA n 306/2004, os RSS acondicionados como sendo do Grupo A, gerados em seis hospitais de Vitória (ES), Brasil, com ênfase na determinação da taxa de geração, do peso específico aparente e da composição gravimétrica dos RSS do Grupo A.

\section{METODOLOGIA}

O estudo foi realizado em seis hospitais, sendo: um público federal e de ensino universitário (H1); um filantrópico e de ensino universitário (H2); um particular (H3); um filantrópico, de referência em oncologia (H4); um público estadual militar (H5) e um público estadual, de referência em traumatologia e ortopedia (H6). Cinco deles são de grande porte, pois possuem mais de 150 leitos e um é de médio porte (H5) por possuir 124 leitos, todos com PGRSS implantados. Foi informado pela Secretaria Estadual de Saúde (SESA), no entanto, que nenhum deles possui PGRSS aprovado, pois não há essa exigência no Estado.

A pesquisa foi desenvolvida entre março de 2008 e julho de 2009. Para a abertura dos sacos de acondicionamento, obteve-se autorização formal do Instituto Estadual de Meio Ambiente e Recursos Hídricos (IEMA), do Comitê de Ética de cada hospital e da empresa responsável pela destinação final, a Central de Tratamento de Resíduos (CTR).

A CTR é composta por um aterro sanitário, um aterro específico para RSS e um incinerador, licenciados pelo IEMA. As características do incinerador são as seguintes: tipo linear de câmaras múltiplas fixas, sem grelha e com sistema de tratamento de gases, resfriamento e filtragem de água, modelo TG 1 X 250/4, 250, kg.h ${ }^{-1}$, de capacidade nominal de $250 \mathrm{~kg} \cdot \mathrm{h}^{-1}$, sendo utilizado como combustível o biogás (metano) filtrado com poder calorífico de 9.800 kcal.(kg.s) ${ }^{-1}$ ou gás natural, diesel e óleo BPF.

As atividades experimentais foram planejadas com base nas informações prestadas pelos responsáveis dos PGRSS de cada hospital, com relação ao atendimento à RDC ANVISA n 306/2004, em questionários e entrevistas. A pesquisa foi desenvolvida sem interferir na rotina do gerenciamento interno e externo dos RSS dos hospitais, exceto na alteração na ordem de coleta.

Os procedimentos adotados para a caracterização dos resíduos de serviço de saúde do Grupo A dos hospitais de Vitória (RSSAHV) foram realizados de acordo com as descrições de Monteiro et al. (2001). Para desenvolvimento de um plano de amostragem em campo, foi realizada uma campanha de amostragem piloto. A partir desta campanha, elaborou-se o procedimento de amostragem, transporte da amostra, recepção da amostra na CTR Marca Ambiental e o número de amostras a serem coletadas, em função das condições operacionais e recursos humanos disponíveis para a execução da tarefa.

A caracterização dos RSS do Grupo A foi realizada entre 15 de junho de 2009 e 11 de julho de 2009, durante 4 semanas, tendo sido gastas 60 horas semanais ao todo. Realizaram-se 12 coletas ao todo, sendo 2 dias de coleta de amostra em cada hospital, sem que acontecesse na semana consecutiva e nem coincidisse com os mesmos dias da semana. Contou-se com o apoio dos hospitais em termos de pessoal e instalações, como o abrigo externo. Em cada campanha foi quantificado o número de sacos brancos leitosos gerados por dia, dos quais foi retirada, aleatoriamente, uma amostra de 14 sacos para avaliação de seus conteúdos. O critério adotado para escolha dos sacos foi o de ele estar com pelo menos $50 \%$ do volume preenchido. Obteve-se 12 amostras de RSSAHV por hospital, com duplicata. A amostra total avaliada foi de 168 sacos, cerca de $9 \%$ de um total de 1.884 sacos gerados nos 12 dias de campanha.

No desenvolvimento do experimento, foram tomados os cuidados necessários para garantir a representatividade das amostras e a integridade física quali-quantitativa destas em relação às características na fonte de geração e ao total de resíduos gerados nos dias de campanha. O transporte dos RSS do Grupo A, desde o abrigo externo de cada hospital até a CTR, foi realizado em veículos específicos da coleta de rotina. Para que não houvesse extravio, rompimento dos sacos de acondicionamento e a prensagem das amostras, durante o transporte acompanharam-se as operações de armazenamento no abrigo externo, a coleta e acomodação das amostras em bags de $900 \mathrm{~L}$, identificados, no veículo coletor. A descarga dos bags foi realizada na área de recepção do incinerador na CTR.

A metodologia de obtenção das amostras compostas de RSS foi elaborada, com certas adaptações, segundo as recomendações delineadas na NBR 10007 (ABNT, 2004), sendo retirados sacos de três seções: abertura, do meio e do fundo do bag. Os RSS foram segregados e classificados por Grupos e subgrupos, segundo a RDC ANVISA nº 306/2004. Após pesagem, eles foram incinerados na própria CTR.

A caracterização foi realizada em ambiente reservado quanto ao acesso de estranhos e adequado quanto à higiene e segurança. Foram utilizados equipamentos como: tesoura para abertura dos 
sacos, balanças digitais com precisão em gramas e em miligramas e recipientes previamente identificados para acondicionar os componentes de RSS segregados por Grupos e subgrupos. Ao término de cada campanha foi feita a higienização e limpeza pessoal, dos equipamentos e do ambiente.

Para facilitar a identificação das amostras durante triagem e análise dos RSS segregados, os subgrupos A1 e A4 foram divididos em A1-1 a A1-5 e A4-1 a A4-17, respectivamente. Foram determinadas as composições gravimétricas em peso para os resíduos do Grupo A - biológicos (RSSA); Grupo B - químicos (RSSB); Grupo D - comuns (RSSD) e da mistura de RSS coletada como Grupo A. Apenas para os RSSAHV foram determinados o peso específico aparente e a taxa de geração por leito ocupado por dia.

A partir das características dos RSS e do conhecimento dos hospitais participantes, os setores, prováveis fontes de geração de RSS do Grupo A, foram identificados por siglas e números: Pronto socorro, PS (1); Centro de Tratamento Intensivo, CTI (2); Ambulatório, AMB (3), Enfermarias, ENF (4), Centro Cirúrgico, CC (5); Unidade Coronariana, UC (6); Banco de Sangue, BS (7); Laboratório, LAB (8); Serviços de Hemodiálise, $\mathrm{H}$ (9); Núcleo de doenças infecciosas, NDI (10); Setor de Higiene e Limpeza, HL (11); Setor Administrativo, ADM (12); Serviço de Copa e Refeitório, CR (13); Posto de Enfermagem, PE (14).

Os dados foram organizados em uma planilha do Excel e se calculou os indicadores apropriados: quantidade média em peso $(\mathrm{kg})$ dos Grupos A, B, D e dos subgrupos A1 e A4; percentual do subgrupo A1 dentro do total do Grupo A (tA); percentual do subgrupo A1 dentro do total da mistura de resíduos (TAHV); percentual do subgrupo A4 dentro do tA; percentual do subgrupo A4 dentro do TAHV; percentual do Grupo B dentro do TAHV; percentual do Grupo D dentro do TAHV; taxa de geração e peso específico aparente. Foram calculadas médias e desvios padrões, para caracterizar os resultados estatisticamente.

\section{RESULTADOS E DISCUSSÃO}

\section{Características do gerenciamento}

Nos 6 hospitais avaliados são realizados, por dia, uma média de 3.347 atendimentos em geral, 1.227 ambulatoriais, 103 internações e 61 cirurgias. Quanto ao gerenciamento dos RSS, todos geram resíduos infectantes e os segregam como RSSA, mas nenhum deles segrega por subgrupos (A1, A2, A3, A4 e A5). Somente um registra as quantidades de sacos de RSSA e RSSD geradas por dia. Em média, são 194 sacos de RSSA e 233 sacos de RSSD. Todos reconheceram gerar RSSB. Dois hospitais, $\mathrm{H} 2$ e $\mathrm{H} 3$, disseram realizar pré-tratamento obrigatório do subgrupo A1. Em três hospitais, H3, H4 e H6, havia identificação dos sacos com rótulos, símbolos e expressões, e apenas um, H4, utilizava sacos de cor laranja para acondicionar os RSSB.

Pela observação visual, independentemente se os RSSA foram pré-tratados ou não, percebeu-se que todos os hospitais os acondicionavam em sacos brancos leitosos a serem enviados para a incineração, e os RSSD em sacos pretos a serem enviados para o aterro sanitário. Essa percepção foi confirmada quando da abertura dos sacos na CTR para segregação.

\section{Taxa de geração e peso específico aparente}

Na Tabela 1 apresenta-se o perfil operacional e de gerenciamento de RSS de cada hospital.

A taxa de geração de resíduos foi $(2,68 \pm 1,41) \mathrm{kg}$.(leito.ocupado.dia $)^{-1}$, variando entre 1,37 a 5,34 kg.(leito.ocupado.dia $)^{-1}$ e o peso específico aparente 106,2 \pm 15 kg.m ${ }^{-3}$, variando entre 101,1 a 108,9 kg.m ${ }^{-3}$.

\section{Composição gravimétrica}

A seguir é feita uma análise da classificação dos RSS à luz de cenários de destinação final, com relação à redução de custos e replanejamento para a melhoria dos PGRSS dos hospitais. Nas Tabelas 2 a 5, têm-se os resultados, em percentagem, das composições gravimétricas dos RSSA, RSSB, RSSD, e RSSAHV (resíduos misturados com RSSA), os respectivos componentes, suas fontes de geração e pesos em valores médios.

Em relação aos resíduos A1-1 a A-5 classificados nesta pesquisa, $60 \%$ são gerados no NDI; $40 \%$ no PS, CTI, AMB, EF, UC; $20 \%$ no CC, BS e $\mathrm{HD}$ e $0 \%$ nos ADM, CR, PE. Dessas fontes, 36\% geram A1-3; 14\% A1-1, A1-4 e A1-5 e 7\% A1-2. Ressalta-se que os resíduos A1-1 representam $11,32 \%$ do total dos RSSA e $4,67 \%$ do TAHV e que os demais (A1-2, A1-3, A1-4 e A1-5) representam apenas 3,14\% de RSSA e $1,29 \%$ TAHV.

Tabela 1 - Taxa de geração e peso específico aparente dos resíduos de serviços de saúde do Grupo A dos hospitais de Vitória por hospital.

\begin{tabular}{|c|c|c|c|c|c|c|c|}
\hline Hospital & $\mathrm{H}^{+}$ & $\mathrm{H}^{+}+$ & $\mathrm{H}^{+}$ & $\mathrm{H} 4^{+}$ & $\mathrm{H} 5^{++}$ & $\mathrm{H}^{+}$ & HV \\
\hline Média do Nº de sacos brancos gerados/dia & 328 & 132 & 126 & 167 & 43 & 146 & 942 \\
\hline Quantidade média (kg.dia') & 1074,6 & 340,5 & 394,6 & 551,2 & 118,8 & 405,1 & 2884,8 \\
\hline Peso específico aparente $\left(\mathrm{kg} \cdot \mathrm{m}^{3}\right)$. & 108,9 & 101,1 & 109,4 & 110,5 & 105,6 & 101,5 & $106,2 \pm 15$ \\
\hline Média de leitos ocupados por dia* & 200 & 180 & 181 & 180 & 86 & 183 & 1010 \\
\hline Taxa de geração (kg por leito-ocupado.dia) & 5,34 & 1,89 & 2,16 & 3,05 & 1,37 & 2,25 & 2,68 \\
\hline
\end{tabular}

*Dados de 2009 disponibilizados pelos hospitais avaliados; RSS: resíduos de serviços de saúde; H1: público federal e de ensino universitário; H2: filantrópico e de ensino universitário; H3:

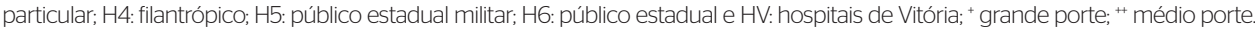


Segundo consta na RDC ANVISA no 306/2004, os resíduos A1 devem ser acondicionados em sacos vermelhos e submetidos a tratamento obrigatório antes da disposição final, dentro ou fora da unidade geradora, conforme o tipo. Os resíduos A1-1 que haviam sido pré-tratados (autoclavação nível III) e sem descaracterização, podem ser destinados em aterro sanitário.
No entanto, verificou-se que todos os resíduos A1-1, independentemente de terem sido tratados nos hospitais, foram enviados para a incineração, acarretando um aumento de custos. Observou-se que ocorreu o mesmo procedimento com resíduos A1-2. Os resíduos A1-3 eram provenientes de hospitais públicos e, de um modo geral, a vacinação não é uma atividade de rotina nos hospitais.

Tabela 2 - Composição gravimétrica média dos resíduos de serviços de saúde do subgrupo A1 de seis hospitais de Vitória, Espírito Santo, em 2009.

\begin{tabular}{|c|c|c|c|c|c|c|}
\hline RSS & Componentes & Fontes Geração & Méd. (kg) & $\mathrm{DP}$ & $\mathrm{A} 1 / \mathrm{tA}(\%)$ & A1/TAHV (\%) \\
\hline A1-1 & $\begin{array}{l}\text { Sobras de amostras de laboratório contendo sangue ou } \\
\text { líquidos corpóreos. }\end{array}$ & 8 e 10 & 1,95 & 3,40 & 11,32 & 4,67 \\
\hline A1-2 & $\begin{array}{l}\text { Bolsas transfusionais, contendo sangue ou hemocom- } \\
\text { ponentes. }\end{array}$ & 7 & 0,19 & 0,46 & 1,10 & 0,45 \\
\hline A1-3 & Vacinas com micro-organismos vivos ou atenuados. & 1 a 4,6 & 0,17 & 0,37 & 0,99 & 0,41 \\
\hline A1-4 & Meios de Cultura & 8,10 & 0,15 & 0,42 & 0,87 & 0,36 \\
\hline A1-5 & $\begin{array}{l}\text { Culturas e estoques de micro-organismos, instru- } \\
\text { mentos para transferência, inoculação ou misturas } \\
\text { de culturas. }\end{array}$ & 8,10 & 0,03 & 0,05 & 0,17 & 0,07 \\
\hline Total A1 & Mistura de RSS (tA1+tA4) & & 2,49 & 3,83 & 14,46 & 5,96 \\
\hline
\end{tabular}

RSS: resíduos de serviços de saúde, Med: Média em peso das 12 amostras diárias avaliadas; DP: Desvio Padrão; tA1: total de A1; tA: total de Grupo A=(tA1+tA4)=17,22 kg.dia'; TAHV: total da mistura de resíduos $(\mathrm{t} A+\mathrm{t} \mathrm{D}+\mathrm{tB})=2884,8 \mathrm{~kg}$.dia'?

Tabela 3 - Composição gravimétrica média dos resíduos de serviços de saúde do subgrupo A4 de seis hospitais de Vitória, Espírito Santo, em 2009.

\begin{tabular}{|c|c|c|c|c|c|c|}
\hline RSS & Componentes & $\begin{array}{l}\text { Fontes } \\
\text { Geração }\end{array}$ & Méd. (kg) & DP & $\mathrm{A} 4 / \mathrm{tA}(\%)$ & A4 / TAHV (\%) \\
\hline A4-1 & Gaze, algodão, atadura & $1 \mathrm{a} 10,14$ & 3,93 & 1,60 & 22,82 & 9,41 \\
\hline A4-2 & Luvas de látex & $1 \mathrm{a} 10,14$ & 3,83 & 0,97 & 22,24 & 9,17 \\
\hline A4-3 & Sacos plásticos de acondicionamento de RSS & $1 \mathrm{a} 10,14$ & 2,13 & 0,74 & 12,37 & 5,10 \\
\hline A4-4 & Peças descartáveis de EPI (capotes, gorros, máscaras) & $1 \mathrm{a} 10,14$ & 1,82 & 1,19 & 10,57 & 4,36 \\
\hline A4-5 & Seringas descartáveis. A4 & 1 a 10,14 & 0,62 & 0,18 & 3,60 & 1,48 \\
\hline A4-6 & Kits de linhas arteriais, endovenosas e dialisadores & $\begin{array}{c}1,2 \\
4 \mathrm{a} 6 \\
9\end{array}$ & 1,43 & 0,91 & 8,30 & 3,42 \\
\hline A4-7 & $\begin{array}{l}\text { Sobras de amostras de laboratório e seus recipientes, } \\
\text { contendo fezes, urina e secreções }\end{array}$ & 8 e 10 & 0,36 & 0,65 & 2,09 & 0,86 \\
\hline A4-8 & Sistema coletor de urina (sistema fechado) & 1 a 9 & 0,24 & 0,40 & 1,39 & 0,57 \\
\hline A4-9 & $\begin{array}{l}\text { Bolsas transfusionais vazias ou com volume residual } \\
\text { pós-transfusão }\end{array}$ & $\begin{array}{c}1,2 \\
4 \text { a } 6 \\
9\end{array}$ & 0,07 & 0,09 & 0,41 & 0,17 \\
\hline A4-10 & Sistema coletor de urina (aberto), uropen & $\begin{array}{c}1,2 \\
4 \text { a } 6 \\
9\end{array}$ & 0,08 & 0,13 & 0,46 & 0,19 \\
\hline A4-11 & Tampas de tubo de ensaio & 8,10 & 0,04 & 0,09 & 0,23 & 0,10 \\
\hline $\mathrm{A} 4-12$ & Cateter nasogástrico & 1 a 6,9 & 0,04 & 0,03 & 0,23 & 0,10 \\
\hline A4-13 & Umidificador com traqueia plástica & 1 a 6,9 & 0,04 & 0,05 & 0,23 & 0,10 \\
\hline A4-14 & Dreno sistema fechado & 1 a 6,9 & 0,05 & 0,10 & 0,29 & 0,12 \\
\hline A4-15 & Tubo orotraqueal & 1 a 6,9 & 0,02 & 0,01 & 0,12 & 0,05 \\
\hline A4-16 & Instrumentos de cirurgia (cateteres e fios guias) & 1 a 6,9 & 0,02 & 0,04 & 0,12 & 0,05 \\
\hline A4-17 & Aspirador cavidade oral & 1 a 6,9 & 0,01 & 0,03 & 0,06 & 0,02 \\
\hline Total A4 & Total dos resíduos A4 & & 14,73 & 3,55 & 85,54 & 35,27 \\
\hline Total A & Total dos resíduos do Grupo A (A1+A4) & & 17,22 & 4,03 & 100,00 & 41,24 \\
\hline
\end{tabular}

RSS: resíduos de serviços de saúde; Med: Média em peso das 12 amostras diárias avaliadas; DP: Desvio Padrão; tA1: total de A1 (Tabela 3); tA4: total de A4; tA: total de Grupo A (tA1+tA4); TAHV: total da mistura de resíduos (tA+tD+tB)=2.884,8 kg.dia- 
Tabela 4 - Composição gravimétrica média dos resíduos de serviços de saúde do Grupo B - embalagens, de seis hospitais de Vitória, Espírito Santo, em 2009.

\begin{tabular}{|c|c|c|c|c|c|c|}
\hline RSS & Componentes & Fontes Geração & Méd. (kg) & DP & $\mathrm{B} / \mathrm{tB}(\%)$ & B/TAHV (\%) \\
\hline B-1 & Medicamentos & $\begin{array}{c}1 \text { a } 6 \\
9,14\end{array}$ & 0,61 & 0,27 & 91,04 & 1,46 \\
\hline tB & Total dos RSS do Grupo B & & 0,67 & 0,27 & 100,00 & 1,60 \\
\hline
\end{tabular}

RSS: resíduos de serviços de saúde; Med: Média em peso das 12 amostras diárias avaliadas; DP: Desvio Padrão; tB: total de B; TAHV: total da mistura de resíduos (tA+tD+tB) = 2.884,8 kg.dia'!

Tabela 5 - Composição gravimétrica média dos resíduos de serviços de saúde do Grupo D, de seis hospitais de Vitória, Espírito Santo, em 2009.

\begin{tabular}{|c|c|c|c|c|c|c|}
\hline RSS & Componentes & Fontes Geração & Méd. (kg) & DP & $\mathrm{D} / \mathrm{tD}(\%)$ & D/TAHV (\%) \\
\hline D-1 & Papel sanitário, fralda, absorventes higiênicos. & $1 \mathrm{a} 14$ & 8,45 & 1,92 & 35,40 & 20,23 \\
\hline D-2 & Equipamentos de soro e similares & $\begin{array}{c}1,2, \\
4 a 6,14\end{array}$ & 6,50 & 3,25 & 27,23 & 15,57 \\
\hline D-3 & Resíduos de área administrativa & $\begin{array}{c}1,2, \\
4 a 6,14\end{array}$ & 5,75 & 1,01 & 24,09 & 13,77 \\
\hline D-4 & Resto alimentar & $\begin{array}{c}1,2, \\
4 a 6,14\end{array}$ & 2,23 & 0,62 & 9,34 & 5,34 \\
\hline D-5 & Resíduos de gesso & 1,3 e 4 & 0,90 & 0,95 & 3,77 & 2,16 \\
\hline D-6 & Espátula de madeira & $\begin{array}{c}1 \mathrm{a} 6, \\
9,10,14\end{array}$ & 0,01 & 0,01 & 0,04 & 0,02 \\
\hline D-7 & Máscara ventilação mecânica de ambu & 1 a 9,14 & 0,01 & 0,02 & 0,04 & 0,02 \\
\hline D-8 & Marca-passo transcutâneo & $1,2,5,6$ & 0,01 & 0,02 & 0,04 & 0,02 \\
\hline D-9 & Sistema de coagulação (oblação cardíaca) elétrico & $1,2,5,6$ & 0,01 & 0,02 & 0,04 & 0,02 \\
\hline tD & Total dos RSS do Grupo D & & 23,87 & 3,52 & 100,00 & 57,16 \\
\hline TAHV & Mistura de resíduos (tA+tD+tB) & & 41,76 & 4,13 & 100.00 & 100,00 \\
\hline
\end{tabular}

RSS: resíduos de serviços de saúde; Med: Média em peso das 12 amostras diárias avaliadas; DP: Desvio Padrão; tD: total de D; TAHV: total da mistura de resíduos (tA+tD+tB).

Essa ocorrência, porém, pode ser atribuída a situações específicas como a necessidade de imunização do quadro de funcionários e/ou de pacientes internados. Considerando que os hospitais H1, H4, H5 e H6 não possuem sistema de tratamento interno, o envio dos resíduos A1-3 para serem incinerados na CTR atende à RDC ANVISA n 306/2004, porém deveriam estar acondicionados em sacos vermelhos e não em sacos brancos leitosos. Dentre os resíduos A1-4 e A1-5, foram identificadas placas de Petri e tubos de ensaio retorcidos, evidenciando terem sido pré-tratados no hospital $\mathrm{H} 1$, o qual disse não realizar pré-tratamento.

Em princípio, o gerenciamento desses resíduos deve ser semelhante ao dado aos resíduos A1-1 e A1-2. Esses resíduos, ao serem acondicionados em sacos brancos leitosos, atendem à RDC ANVISA $n^{\circ} 306 / 2004$, porém, quanto à disposição final, eles poderiam ser dispostos em aterro específico de RSS da CTR, evitando-se o custo elevado da incineração.

Os RSSA4, pela RDC ANVISA n 306/2004, podem ser dispostos, sem tratamento prévio, em local licenciado. Eles representaram 87,54\% dos resíduos coletados como sendo do Grupo A, e 35,27\% do TAHV. Desses resíduos, 77\% são gerados nos PS e CTI; 73\% nos EF, CC e UC; $68 \%$ no NDI; $64 \%$ no LAB; $50 \%$ nos AMB; $45 \%$ no BS;
$22 \%$ nos PE e $0 \%$ nos HD, HL, ADM e CR. Dessas fontes, 79\% geram A4-1, A4-2, A4-3, A4-4 e A4-5; 57\% A4-8, A4-9, A4-10 e A4-12; 50\% A1-2; 43\% A4-6, A4-13, A4-15, A4-16 e A4-17; 36\% A1-3; 21\% A4-14; 14\% A4-1, A1-4, A1-5, A4-7 e A4-11.

Os resíduos A4-3, de modo geral, não são provenientes das atividades hospitalares, tratavam-se somente de sacos vazios de acondicionamento de RSS. Alguns estavam abertos, possivelmente, por terem sido utilizados como camada impermeabilizante de leitos, evitando a infiltração de fluidos corporais nos lençóis e outros foram esvaziados na pesquisa. Os resíduos A4-4 são resultantes do processo de assistência direta à saúde, que pela alta probabilidade de terem entrado em contato com sangue e/ou líquidos corpóreos, foram classificados como RSSA4.

Dentre os resíduos A4-5, foram encontradas seringas sem agulhas acopladas, contendo volume residual ou vestígios de sangue e/ou outras substâncias que não foram possíveis de ser identificadas, e por isso foram classificadas como resíduos A4. As seringas podem também ser resultantes de atividades de vacinação com micro-organismos vivos ou atenuados devendo, de acordo com a RDC ANVISA n 306/2004, ser classificadas como A1. Dentre os resíduos A4-6, foram encontrados kits de linhas arteriais, endovenosas, dialisadores e equipos de soro. Considerando que a maioria dos equipos e dialisadores estava 
contaminada com sangue não na forma livre, eles foram classificados como RSSA4. Os resíduos A4-7 são os gerados em laboratório que, devido à forma com que foram acondicionados, indicam ser provenientes de pacientes que não continham e nem eram suspeitos de conter agentes Classe de Risco IV, sem relevância epidemiológica e risco disseminação ou micro-organismo causador de doença emergente que se torne epidemiologicamente importante ou cujo mecanismo de ação seja desconhecido. No entanto, devido à dificuldade para identificar se houve ou não contaminação e/ou inexistência de tratamento interno dos resíduos A4-7, nesta pesquisa a destinação indicada desses resíduos seria a incineração. Quanto aos resíduos A4-8 a A4-17, por serem provenientes de atividades de assistência à saúde e por não conterem sangue ou líquidos corpóreos na forma livre e serem de fontes de geração semelhantes, foram classificados como resíduos A4.

Os RSSB-1 foram os presentes em maior percentagem dentre o Grupo B. São gerados em vários setores e, especificamente, nas farmácias e nos postos de enfermagem; os RSSB-2 são provenientes de atividades de desinfecção, principalmente, de instrumentos e equipamentos, podendo ser embalagens de produtos químicos específicos para o uso hospitalar, representando maior risco à saúde pública e/ou ao meio ambiente, se mal destinados. Se os RSS do Grupo B não estivessem misturados aos do Grupo A, poderiam até ser reutilizados como embalagens de produtos semelhantes. Além disso, mesmo em pequenas quantidades, merecem cuidados especiais, podendo ser dispostos, segundo a RDC ANVISA n 306/2004, em aterro sanitário e/ou aterro específico para RSS, não precisando ser incinerados.

No Grupo D, os subgrupos D-1 a D-9, classificados nesta pesquisa, foram encontrados misturados aos RSSA, em todos os setores: $100 \%$ no PS; $89 \%$ no CTI, CC e UC; $77 \%$ nas ENF; $67 \%$ nos PE; $45 \%$ nos AMB; $33 \%$ nos HD; $22 \%$ no BS, LAB e NDI; e $11 \%$ no HL, ADM e CR. Dessas fontes, $100 \%$ geram D-1; 71\% D-7; 63\% D-6; 36\% D-2, D-3 e D-4; 22\% D-5. Os D-1 foram os presentes em maiores percentuais, gerados em todos os setores dos hospitais, principalmente nos banheiros e lavatórios. O papel toalha, comumente gerado após assepsia das mãos, não oferece riscos biológicos; as fraldas descartáveis e os absorventes higiênicos, se forem originários de pacientes infectados com micro-organismos de Classe de Risco IV, deveriam estar em sacos vermelhos e não nos sacos brancos avaliados. Devido à impossibilidade de identificar se houve ou não contaminação desses resíduos na origem, todos foram considerados como RSSD. Os resíduos D-2 são gerados, principalmente, nos PS, CTI, CC, UC, PE, ENF; os resíduos D-3 são gerados em todos os setores dos hospitais, principalmente nos ADM; os D-4 têm como fonte principal as ENF, no entanto, podem ter sido gerados em todos os setores dos hospitais, uma vez que eles possuem copa para atender o quadro funcional. Os resíduos D-5 são gerados nos PS, AMB, ENF, principalmente nos serviços de ortopedia. Os resíduos D-8 são gerados nos setores de urgência e emergência, PS, CTI, CC e UC, considerados como RSS do Grupo D por não serem equipamentos invasivos, entrando apenas em contato com a pele. Os resíduos D-9 não são materiais descartáveis, sendo reutilizado após descontaminação. Possivelmente apresentaram defeitos e, por essa razão, foram descartados. Os RSSD encontrados, caso não estivessem misturados com os RSSA, deveriam, segundo a RDC ANVISA n 306/2004, estar acondicionados em sacos plásticos para resíduos comuns, podendo ser dispostos em aterro sanitário devidamente licenciado, ao invés de serem incinerados, com significativa redução de custos em relação à incineração.

Os RSS do grupo E encontrados nos sacos brancos foram: quinze agulhas e três lancetas sem proteção retrátil, com peso médio total de 0,04 kg. Do ponto de vista de prevenção de acidentes ocupacionais, nenhuma unidade devia ter feito esse tipo de descarte. Os resíduos perfuro-cortantes devem ser acondicionados em recipientes rígidos, apropriados para conter o risco.

\section{Avaliação da taxa de geração}

Nesta pesquisa, a taxa de geração dos resíduos misturados (TAHV) obtida foi de 2,68 kg.(leito.ocupado.dia) ${ }^{-1}$, sendo $1,15 \mathrm{~kg}$ de resíduos do Grupo A. Cussiol, Lange e Ferreira (2001) obtiveram, em um hospital pediátrico de Belo Horizonte, a taxa de 1,28 kg.(leito.ocupado.dia) ${ }^{-1}$. Estes dois últimos valores são muito próximos, muito embora as formas de obtenção fossem bem diferentes.

Cussiol, Lange e Ferreira tiveram acesso às fontes de geração e treinou os geradores para fazer o descarte correto dos resíduos. Já nesta pesquisa, a segregação foi na CTR, sem nenhum envolvimento com os geradores, inclusive sem avisar previamente o dia da coleta. Acredita-se, portanto, que se a metodologia de Cussiol, Lange e Ferreira fosse adotada neste estudo os resultados também seriam semelhantes.

Salomão, Trevizan e Günther (2004) obtiveram, em Centro Cirúrgico (CC) de dois hospitais da cidade de São Paulo, taxas de geração de RSS variando de 0,93 a 1,91 kg, com valor médio de 1,42 kg por cirurgia.dia ${ }^{-1}$. Souza (2005) obteve, em um hospital de grande porte da cidade Jaú, São Paulo, especializado em oncologia, uma taxa de geração de RSS de 1,88 kg por leito.dia-1 ${ }^{-1}$ dos quais $62 \%$ são infectantes, resultando numa taxa de RSSA de 1,17 kg.(leito.ocupado.dia) ${ }^{-1}$, valor semelhante aos $1,15 \mathrm{~kg}$.(leito.ocupado.dia) ${ }^{-1} \mathrm{de}$ RSSA aqui encontrados.

Gil (2007) obteve, no hospital da Irmandade Santa Casa de Misericórdia de São Carlos (SP), o valor de 1,21 kg por leito.dia ${ }^{-1}$, e concluiu que esse é um valor aceitável e menor do que os encontrados por outros pesquisadores no Brasil. Portanto, ao se fazer uma correta segregação na fonte nos hospitais de Vitória, é possível minimizar a taxa de geração de resíduos do Grupo A de 2,68 para $1,15 \mathrm{~kg}$.(leito.ocupado.dia) ${ }^{-1}$. 
A taxa de RSSA dos hospitais participantes da pesquisa encontra-se dentro da média de geração nacional, considerando o mesmo porte e perfil administrativo de atendimento. Cheng et al. (2003), em um estudo sobre RSS em hospitais de Taiwan, obteve uma variação da taxa de 0,44 a 0,88 kg.(leito.dia) ${ }^{-1}$ para resíduos infectantes, e Diaz et al. (2007), em um estudo sobre a variação da geração dos RSS em Ulaanbaatar, Mongólia, encontrou taxas de 0,016 a 3,23 kg.(leito.dia) ${ }^{-1}$, tanto para RSS do Grupo A quanto em frações misturadas. A taxa de geração obtida nesta pesquisa está dentro de uma média internacional, porém, considerando as diferentes legislações e formas de classificação dos RSS em outros países, não é possível fazer uma comparação representativa.

\section{Avaliação do peso específico aparente}

Dentre os principais fatores que interferem na determinação do peso específico aparente dos RSS têm-se: a variedade dos tipos de resíduo (plásticos, papeis, vidros, borracha, panos, materiais orgânicos, fraldas descartáveis usadas, secreções, fluidos em geral); a acomodação dos resíduos no interior dos sacos e o grau de compactação. O descarte de líquidos em lixeiras, embora não seja uma prática correta, é muito comum em algumas unidades de saúde, resultando no aumento da umidade e do peso dos RSS.

O peso específico aparente obtido para os resíduos misturados (RSSAHV) foi $(106,2 \pm 15) \mathrm{kg} \cdot \mathrm{m}^{-3}$, valor bem inferior aos $280 \mathrm{~kg} \cdot \mathrm{m}^{-3}$ recomendado por Monteiro et al. (2001), na ausência de dados precisos, e aos $226 \mathrm{~kg} \cdot \mathrm{m}^{-3}$ e $262 \mathrm{~kg} \cdot \mathrm{m}^{-3}$ citados por Diaz et al. (2007), referentes a hospitais do Peru e das Filipinas, respectivamente. Uma das prováveis razões dessas diferenças pode ter sido a ausência dos RSS do subgrupo A3 - peças anatômicas do ser humano, produto de fecundação sem sinais vitais —, na composição gravimétrica dos RSSAHV. Segundo Diaz et al. (2007), o peso específico depende da umidade e das dimensões dos RSS, parâmetros importantes para se projetar e operar sistemas de disposição final (aterros) e de tratamento de RSS, como os incineradores.

\section{Avaliação de custo de destinação dos resíduos}

Segundo a RDC ANVISA n 306/2004, todos os resíduos A4, D e B encontrados nesta pesquisa poderiam ter sido dispostos, sem tratamento prévio, em aterro específico de RSS ou sanitário devidamente licenciados, com redução de custos em relação à incineração ora praticada.

Com base na taxa de geração 2,68 kg.(leito.ocupado.dia) ${ }^{-1}$, foi estimada uma geração de 1.038,48 t.ano ${ }^{-1}$ de RSSAHV no ano de 2009. Havendo segregação apropriada dos 57,16\% de RSSD encontrados acondicionados junto com o RSSA, a taxa de geração de RSSA passaria a ser de 1,15 kg.(leito.ocupado.dia) ${ }^{-1}$ ou 446,57 t.ano ${ }^{-1}$.
Se as 591,96 t.ano-1 de RSSD restantes fossem aterradas na CTR, o custo anual total com incineração e aterro resultaria numa redução de custos de 57,71\%, com base nos valores informados pela CTR.

Havendo, ainda, tratamento interno obrigatório dos resíduos A1 nos seis hospitais e o acondicionamento apropriado dos 34,41\% de RSSA4, com exceção dos RSSA4 -7 e dos 1,6\% de RSSB para os quais a incineração foi considerada adequada, o percentual de RSSA a ser incinerado seria de apenas $6,82 \%$. Nesse caso, a taxa de geração passaria a ser de $0,18 \mathrm{~kg}$.(leito.ocupado.dia) ${ }^{-1}$, ou 70,56 t.ano-1 de resíduos a serem incinerados, e taxa de geração de $2,50 \mathrm{~kg}$.(leito.ocupado.dia) ${ }^{-1}$, ou 967,92 t.ano $^{-1}$ de resíduos a serem dispostos em aterro especial ou sanitário. A segregação dos resíduos e a melhor escolha dos sistemas de destinação resultariam em uma economia de $93 \%$ nos gastos, quando comparado com o modelo vigente.

\section{Análise da gestão dos resíduos}

Frente aos resultados avaliados, para que ocorra a melhoria contínua do gerenciamento dos resíduos nos hospitais avaliados, cabe aos órgãos de controle ambiental, federal, estadual e municipal como a ANVISA, o IEMA, a Secretaria Estadual de Saúde (SESA) e a Secretaria de Meio Ambiente da Prefeitura Municipal de Vitória (ES), não apenas licenciar as Unidades de Tratamento e Destinação final com base na Resolução CONAMA n 358/2005, e fazer checklist sobre os PGRSS dos hospitais, como nos foi informado pela SESA.

Cabe a esses órgãos promover, também, o desenvolvimento de práticas sustentáveis e conscientização que resultem na minimização de gastos desnecessários com incineração e que possibilitem a redução de custos com disposição final.

O fato é que no Estado do Espírito Santo o único incinerador de RSS existente já se encontra com sua capacidade operacional totalmente ocupada com os RSS de um único município e incinerando resíduos que poderiam ser aterrados, enquanto em alguns municípios do estado, os RSS, que obrigatoriamente deveriam ser enviados para incineração ou para ser aterrados na CTR, continuam sendo dispostos em depósitos irregulares.

\section{Proposta para planejamento de melhorias no gerenciamento dos resíduos}

Partindo do princípio de que as falhas de segregação dos RSS podem ser reduzidas com a implantação de um sistema de gestão preconizado por órgãos de controle ambiental (federal, estadual e municipal), aponta-se a necessidade de uma melhor segregação na fonte de RSS que minimize as condições de riscos ambientais e ocupacionais: a segregação na fonte de RSS do Grupo B e E; a criação de monitoramento periódico de indicadores; fazer com que os gastos com o gerenciamento dos RSS passem a ser custeados pelos hospitais em não pela prefeitura e o real cumprimento das legislações vigentes. Essas ações são suficientes 
para que se tenha uma disposição e destinação adequada, mais segura e econômica, priorizando a saúde pública, a segurança ocupacional e a preservação ambiental.

Apresentam-se, a seguir, sugestões para o gerenciamento dos RSS nos hospitais participantes, visando à redução de custos, riscos e à preservação do meio ambiente. Para redução da mistura dos RSSD, recomendam-se as medidas: educação continuada dos funcionários que enfatize o real conceito dos RSSD; mapeamento dos locais e das quantidades de lixeiras disponíveis para o acondicionamento dos RSSD e RSSA; caso seja necessário, deverá ser aumentada a quantidade de lixeiras nos setores de maior geração de RSSD, como nos de urgência e emergência (PS, CTI, CC, UC). Essas medidas deveriam ser iniciadas numa primeira etapa, por exemplo, nos seis primeiros meses, em setores com maior potencial de geração: PS, CTI, CC, UC e ENF. Numa segunda etapa, os mesmos cuidados devem ser estendidos, pelo mesmo período, aos setores de menor geração: $A M B$, HD, BS, LAB, NDI. Por fim, uma terceira etapa deve envolver de forma rigorosa e direta todas as fontes, inclusive os setores $\mathrm{HL}, \mathrm{ADM}$ e CR, de forma que se obtenha $100 \%$ de segregação na fonte dos 57,16\% de RSSD, atualmente misturados com os RSSA.

Os resíduos dos subgrupos A4-1 a A4-17 representam a maior parte dos RSS enviados para incineração, deveriam ser aterrados e, para redução quantitativa do envio à incineração, recomenda-se a seguinte medida: educação continuada nos setores, abordando todos os funcionários envolvidos no processo de assistência à saúde, com ênfase na diferenciação entre os RSSA1 e os RSSA4. Segundo a RDC, os resíduos A4 devem ser acondicionados em sacos brancos e os A1 não tratados devem ser acondicionados em sacos vermelhos e encaminhados separadamente para tratamento obrigatório interno ou externo, conforme o tipo. Essas medidas deveriam ser iniciadas numa primeira fase, por exemplo, nos setores com maior potencial de geração, PS, CTI, ENF, CC, UC, NDI e LAB; numa segunda etapa, deveriam ser envolvidos os setores BS, PE e numa terceira etapa envolver os demais RSSA4 de forma que se obtivesse $100 \%$ de segregação na fonte e envio para aterro especial e/ou sanitário dos 35,27\% de TAHV.

Para redução da geração dos RSSA1 recomendam-se estas medidas: para os RSS A1-1, A1-4 e A1-5, que são gerados nos laboratórios (LAB e NDI), a execução de rotinas de tratamento interno (autoclavação) e o posterior acondicionamento do resíduo, de acordo com suas características físicas, em RSSD ou RSSA4. Isso seria suficiente para a redução desejada do percentual de A1 dentro dos TAHV.

Da mesma forma, os RSS A1-2, gerados no BS, a rotina de recuperação das bolsas instaladas em que a transfusão fora incompleta, com posterior tratamento dentro do setor, seriam suficientes para redução ainda maior dos resíduos A1. Os resíduos A1-3 são gerados no PS, CTI, AMB, PE e UC. Sugere-se que todos esses setores enviem o resíduo para um único setor de acondicionamento de medicamentos e que dê posterior destinação adequada. Ressalta-se que os resíduos D e A4, com suspeita ou confirmação de contaminação anteriormente descrita, devem ser classificados como A1, e os hospitais deverão oferecer estrutura e material necessários para tratamento interno ou um acondicionamento e transporte seguros até a CTR, compatíveis com o tipo de RSS, que de modo geral não é tratado dentro da unidade.

\section{CONCLUSÕES}

A composição gravimétrica de uma amostra diária de 41,75 kg obtida em doze campanhas e representativa de $2.884,8 \mathrm{~kg}$ foi de: $57 \%$ do Grupo D; $41 \%$ de RSS efetivamente do Grupo A; 1,5\% do Grupo B e 0,05\% do Grupo E. A taxa média de geração foi de $2,68 \mathrm{~kg}$.(leito.ocupado.dia) ${ }^{-1}$ e o peso específico aparente de 106,2 kg.m $\mathrm{m}^{-3}$.

A segregação dos RSS do Grupo D, misturados com o A, reduziria a quantidade de resíduos do Grupo A para 1,15 kg.(leito.ocupado.dia) ${ }^{-1}$, com redução de custos com incineração de $57,71 \%$. Se, além disso, houver segregação por subgrupos e tratamento obrigatório poder-se-ia alcançar uma taxa de geração de $0,18 \mathrm{~kg}$.(leito-ocupado.dia) ${ }^{-1}$ de RSSA; e, se 93,28\% destes forem dispostas em aterro sanitário, sendo 6,82\% incineradas, obtém-se uma redução de custos no gerenciamento de até $93 \%$.

Para melhorar o PGRSS é necessário que os hospitais ofereçam treinamento de pessoal; estrutura física e material para um acondicionamento apropriado para os RSS do subgrupo A1 não tratado; implantação de tratamento interno obrigatório para o subgrupo A1 e que também haja um maior envolvimento dos gestores, do corpo clínico, de enfermagem e demais frequentadores dos hospitais; e ações governamentais, no que diz respeito à disposição final adequada e cumprimento, na íntegra, da RDC ANVISA n 306/2004 e Resolução $n^{\circ} 358 / 2005$ do CONAMA.

\section{AGRADECIMENTOS}

Ao Programa de Pós-Graduação em Engenharia Ambiental da Universidade Federal do Espírito Santo (PPGEA/UFES), aos hospitais participantes do estudo, à Fundação de Amparo a Pesquisa do Espírito Santo (FAPES), à Faculdade de Ciências Sociais e Tecnológicas (FACITEC), à MARCA AMBIENTAL e ao Instituto Estadual de Meio Ambiente (IEMA). 


\section{REFERÊNCIAS}

ABNT - Associação Brasileira de Normas Técnicas. (2004) NBR 10007: Amostragem de resíduos sólidos. Rio de Janeiro, Brasil.

BRASIL. Ministério do Meio Ambiente e da Amazônia Legal. (2005) Resolução CONAMA n ${ }^{\circ}$ 358, de 29 de abril de 2005. Diário Oficial da União, 4 de maio de 2005. Seção 1, p. 63-65.

BRASIL. Ministério da Saúde. Agência Nacional de Vigilância Sanitária. (2004) Resolução da Diretoria Colegiada-RDC n 306, de 7 de dezembro de 2004. Diário Oficial da União, 10 de dezembro de 2004.

ANVISA - Agência Nacional de Vigilância Sanitária. (2006) Manual de gerenciamento de resíduos de serviço de saúde. Brasília, Brasil.

CUSSIOL, N.A.M.; LANGE, L.C.; FERREIRA J.A. (2001) Taxa de Geração de Resíduos de Serviços de Saúde em um Hospital Pediátrico. In: $21^{\circ}$ Congresso Brasileiro de Engenharia Sanitária e Ambiental, Anais ... Belo Horizonte: Associação Brasileira de Engenharia Ambiental.

CHENG, Y.W.; SUNG, F.C.; YANG, Y., LO, Y.H.; CHUNG, Y.T., LI, K.C. (2008) Medical waste production at hospitals and associated factors. Waste Management, v. 29, n. 1, p. 440-444.

DIAZ, L.F., EGGERTH, L.L., ENKHTSETSEG, Sh., SAVAGE, G.M. (2007) Characteristics of healthcare wastes. Waste Management, v. 28, n. 7, p. $1219-1226$
FEAM - Fundação Estadual do Meio Ambiente. (2008) Manual de gerenciamento de resíduos de serviços de saúde/Fundação Estadual do Meio Ambiente. Belo Horizonte, Brasil, 88 p.

GIL, T.N.L. (2007) Caracterização Qualitativa e Quantitativa dos Resíduos de Serviços de Saúde Gerados na Irmandade Santa Casa de Misericórdia de São Carlos, SP. Monografia (Trabalho de Graduação em Engenharia Ambiental) - Universidade de São Paulo, São Carlos (SP), Brasil.

MONTEIRO, J.H.P.; FIGUEIREDO, C.E.M.; MAGALHÃES, A.F.; MELO, M.A.F.; BRITO, J.C.X.; ALMEIDA, T.P.F.; MANSUR, G.L. (2001) Manual de Gerenciamento Integrado de Resíduos Sólidos. Rio de Janeiro: IBAM.

SALOMÃO, I.S.; TREVIZAN, S.D.P.; GÜNTHER, W.M.R. (2004) Segregação de Resíduos de Serviços de Saúde em Centros Cirúrgicos. Engenharia Sanitária Ambiental, v. 9, n. 2, p. 108-111.

SouZA, E.L. (2005) Medidas para Prevenção e Minimização da Contaminação Humana e Ambiental Causada pelos Resíduos de Serviço de Saúde Gerados em Estabelecimento Hospitalar - Estudo de Caso. 145 f. Tese (Doutorado) - Universidade de São Paulo, São Carlos, Brasil. 